\title{
Expert protocol teams enhance patient centered cardiac testing and care
}

\author{
R. G. Schwartz, MD, MS, MASNC, ${ }^{\text {a,b }}$ L. Sculli, BSN, ${ }^{a}$ L. Reinhardt, BSN, ${ }^{a}$

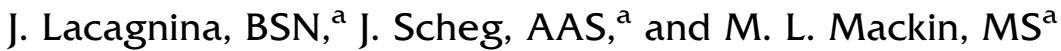 \\ a Cardiology Division, Department of Medicine, The University of Rochester Medical Center, \\ Rochester, NY \\ b Nuclear Medicine Division, Department of Imaging Sciences, The University of Rochester \\ Medical Center, Rochester, NY
}

Received Aug 14, 2017; accepted Aug 14, 2017

doi: 10.1007/s12350-017-1047-0

\section{See related article, pp. 536-540}

The field of nuclear cardiology has witnessed major advances in recent decades which have enhanced the quality and effectiveness of care. New radiotracers, the migration from planar to tomographic imaging, the development cardiac PET, cadmium zinc telluride SPECT, flow quantification with PET and CZT SPECT, $\mathrm{CAD}$ evaluation in the obese and other special populations, evidence based monitoring of therapeutic effectiveness of lifestyle, medical, and revascularization interventions; stress only imaging with ultra low dose radiation exposure afforded by attenuation corrected SPECT MPI, assessment of the risks of cardiotoxicity of modern cancer therapy and prevention of heart failure; molecular imaging of hibernating viable myocardium to assess benefit of revascularization, metabolic assessment of infiltrative diseases including sarcoidosis and amyloidosis, and assessment of heart failure risk and therapeutic response with MIBG, hydroxy ephedrine, and quantification of cardiac dyssynchrony have greatly augmented the number and complexity of studies performed routinely in clinical practice. The proliferation of nuclear cardiology testing options within the field has

Reprint requests: R. G. Schwartz, MD, MS, MASNC, Cardiology Division, Department of Medicine, The University of Rochester Medical Center, Rochester, NY; ronald_schwartz@urmc. rochester.edu

J Nucl Cardiol 2019;26:541-2.

$1071-3581 / \$ 34.00$

Copyright (C) 2017 American Society of Nuclear Cardiology. increased the complexity and sophistication required to order, perform, and interpret nuclear cardiology studies.

With the advances of nuclear cardiology imaging technology comes the responsibility to perform appropriate testing by providing the right test at the right time, or no testing when requested for a specific patient to address the clinical questions posed. The technical complexity of testing options presents a formidable series of challenges for the smooth and efficient operation of the individual nuclear cardiology laboratory. In the current era of rigid and limited sophistication of computerized ordering of testing options, a need clearly exists for the nuclear cardiology laboratory to provide expertise to define the indications and protocols for appropriate testing for each referred patient. However, few practice models have been reported to facilitate patient centered imaging.

In this issue of the Journal, ${ }^{1}$ the nuclear cardiology laboratory of the University of Vermont reports the effects of a change from script driven scheduling to expert nurse-driven protocol selection of stress tests to be reviewed and ordered by the authorized physician users of radioisotopes in the nuclear laboratory. They report the implementation of an expert protocol nurse substantially reduced patient no shows and test cancelations. They found instituting protocol nursing of all non-imaging (ETT) and nuclear (MPI) stress tests $(n=3071)$ resulted in protocol changes in $36 \%$ of their patients, and reduced test cancelations by $73 \%$ and protocol changes by $48 \%$. These changes were sustained over two successive 6 month periods following a baseline observation period of 6 months.

The potential implications of this report are substantial. Review of testing orders by the nurses in the laboratory can be expected to enhance the ratio of appropriate to rarely appropriate studies and enhance the 
cost effectiveness of stress testing in the nuclear cardiology laboratory, as suggested by the authors. Further evaluation of the impact of expert nurse review of protocol orders on the appropriateness category and cost effectiveness of studies performed are useful new hypotheses for testing. These data can help optimize staffing requirements to support expert teams to in stress testing and imaging laboratories to enhance patient centered imaging and quality care.

Is the experience of the University of Vermont team reproducible? We suspect the function and experience of expert teams to optimize patient centered imaging is likely widespread, highly effective, and under-reported. Our experience at the University of Rochester Medical Center is consistent with that reported by the University of Vermont team: The protocol nurse gives telephone instructions prior to the scheduled test which includes review of test indications, medications, dietary instructions, appropriate dress, correct test selection, appointment time, and responds to patient questions. The protocol nurse routinely reduces communication errors that may occur between ancillary staff and providers, and clarifies details with the laboratory director or attending nuclear cardiologist. Our staff nurses and nuclear technologists routinely review indications; identify, evaluate, communicate, and resolve patient safety concerns, document appropriateness indications of testing, and advocate for patient well being and intervene by raising questions to the cardiology fellow in training and the attending nuclear cardiologist regarding conditions which may limit the ability of the patient to be adequately or safely tested. In this way, nurse and technologist experts not only provide sophisticated care needs of the patient, but also serve to reassure patients, enhance patient satisfaction with the nuclear cardiology testing, and provide invaluable training for residents and cardiology fellows in the process. These discussions may lead to changes in the protocol, cancelation of the test, and referral directly to the catheterization laboratory for patients who by virtue of symptoms, ECG findings, or biomarkers suggest an alternative clinical evaluation strategy which may be more appropriate. Thus, these evaluations enhance patient centered imaging, optimize patient safety and clinical effectiveness, and enhance the training experience of the program. In our experience, these expert team processes provide a strong measure of patient centered caring that provides high patient satisfaction.

Many nuclear cardiology laboratories have likely accommodated the proliferation and complexity of testing protocols by utilizing teams of physicians supported by highly expert technologists and nurses to help define and monitor the ordering, scheduling, and performance of appropriate testing on an individual basis. In this era of complex testing, centralization of scheduling, and scrutiny of health care costs, the University of Vermont report on the role of the expert protocol nurse is timely and compelling. Importantly, this report defines an effective evidence based model of patient centered imaging to enhance accuracy, efficiency, appropriateness, and cost effectiveness of cardiac imaging that is worthy of citing and emulating in the best interests of patient care.

\section{Disclosures}

Dr. Schwartz is on the Astellas Speakers Bureau. L. Sculli, L. Reinhardt, J. Lacagnina, J. Scheg, and M. L. Mackina have no disclosures.

\section{Reference}

1. Salimi PN, Niggel JB, Keating FK. How to achieve patient-centered testing: Role of the protocol Nurse. J Nucl Cardiol 2017. doi: 10.1007/s12350-017-0911-2. 